ISSN: 0210-1696

DOI: http://dx.doi.org/10.14201/scero20154625777

\title{
REFLEXIONES SOBRE EL APOYO CONDUCTUAL POSITIVO
}

\section{Reflections on Positive Behavior Support}

\author{
Sebastià Forteza BaUzá \\ Consorcio Aprop. Carr. Valldemossa, 98 (km 2). 07120 Palma de Mallorca (Baleares) \\ sebastia.forteza@aprop.org \\ Vicenç Ferretjans Moranta \\ Mater Misericordiae \\ Sally-Ann Cebrian Tunbridge \\ Aproscom \\ Teresa Font Jaume \\ Amadipesment \\ José E. Vicent Primo \\ Amadiba \\ María R. SAlva ObRador \\ Intress
}

Recepción: 26 de junio de 2014

Fecha de aceptación definitiva: 16 de enero de 2015

Biblid. [0210-1696 (2015) vol. 46 (2), n. o 254, abril-junio; 57-77]

Resumen: El Apoyo Conductual Positivo (ACP) representa un movimiento científico que une dos líneas de trabajo, una bajo el Análisis Conductual Aplicado y otra de carácter teórico que recoge valores y conceptos descritos y desarrollados en el campo de las discapacidades intelectuales. En sus inicios fue una intervención ante las conductas problemáticas con procedimientos positivos en contraposición al abuso de prácticas negativas (aversivas). Paso a paso, se comprobó que las técnicas positivas necesitaban ser aplicadas de forma preventiva y proactiva para educar y reforzar habilidades cuya equivalencia funcional fuera la misma que motivaba las conductas problemáticas. Por último, se introdujeron claves de la línea teórica y del contexto, como calidad, normalización, valores, etcétera. Hoy el ACP ha trascendido su primer cometido y es una forma de trabajar en cualquier servicio independientemente de que haya o no conductas problemáticas, más aún, está siendo aplicado en contextos escolares, 
judiciales y de tercera edad. Ahora bien, el ACP tiene bastantes desafíos, entre otros la definición operativa de los conceptos que caen bajo la vertiente teórica y su desarrollo discriminativo. Por otra parte, para algunos, el abuso del discurso en relación con la falta de concreción operativa y el uso de conceptos indiscutibles (que nadie pondría en duda, como el de positivo) representa un peligro a la credibilidad científica del ACP.

Palabras clave: Apoyo Conductual Positivo; servicios; resultados.

Abstract: Positive Behavior Support (PBS) is a scientific movement that unites two lines of work, one under the Applied Behavioral Analysis and the other of a more theorical type, that collects concepts described and developed in the field of intellectual disabilities. In the beginning it was an intervention addressed to challenging behavior with positive procedures as opposed to the abuse of negative practices (aversive). Step by step, it was found that the positive techniques needed to be applied preventively and proactively to educate and reinforce skills whose functional equivalence is the same as that which motivated the problem behaviors. Finally, keys from a theorical line and context were introduced, such as quality standards, values, and so on. Today PBS has transcended its first task and has become a way to work in any service, regardless of whether or not there are challenging behaviors, furthermore, it's being implemented in school, court and seniors contexts. However, PBS has many challenges, including the operational definition of concepts that fall under the theorical side and it's discriminative development. Moreover, for some, the abuse of speech in relation to the lack of operational realization and use of indisputable concepts (which no one would doubt, such as positive) presents a danger to the scientific credibility of PBS.

KEY wORds: Positive Behavioral Support; services; results.

\section{Introducción}

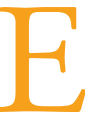

N FEBRERo DEL AÑo 2011 FeAPS Baleares organizó un encuentro con el profesor Edwin Jones, de la Universidad del Sur de Gales, quien explicó la implementación del Apoyo Conductual Positivo (ACP) en los servicios para personas con discapacidad intelectual y conductas problemáticas del país de Gales. Una idea que surgió del encuentro fue la creación de una comisión técnica para el estudio de esta metodología de trabajo y su difusión a toda la red Feaps-Baleares. La presente reflexión es fruto del estudio de la bibliografía consultada.

Así, el ACP une dos líneas: una teórica y otra técnica. En la teórica se agrupan las ideas referidas a la cultura, los valores, los derechos y la ética de los sistemas dirigidos al mundo de personas con discapacidad intelectual. Se implican creencias, visiones, estilos y formas de trabajar que sin vacilar guían los objetivos, es decir, si se defiende un valor todas las acciones emprendidas por los servicios se alinearán con éste.

La parte técnica, más concreta quizá, incluye los aspectos del diseño y procedimientos para llevar a cabo las intervenciones. En definitiva, son las técnicas que caerían bajo el rótulo de Análisis Conductual Aplicado (ACA), siempre y cuando no sean aversivas.

(C) Ediciones Universidad de Salamanca

Siglo Cero, vol. 46 (2), n. ${ }^{\circ}$ 254, 2015, abril-junio, pp. 57-77 
Hoy el ACA diseña programas dirigidos al refuerzo de habilidades, educación de nuevas conductas, generalización, prevención, etcétera.

Entre la línea teórica y la técnica debe existir total coherencia, por ejemplo, si se cree en la autodeterminación, línea teórica, los usuarios deben estar presentes y participar en el diseño de sus planes, línea técnica.

Por tanto, el ACP sería el uso de apoyos técnicos con un compromiso teórico positivo. El adjetivo positivo debe tratarse de dos maneras: una referente al uso de técnicas conductuales no aversivas, como los procedimientos que potencien las habilidades, y otra a los elementos de calidad de vida que afectan al bienestar de la persona.

Debemos resaltar que un uso de procedimientos positivos por sí solo no sería ACP, por ejemplo, un 'laissez faire' argumentando respetar a la persona no podría ser una práctica positiva si detrás no existe un planteamiento técnico. Lo contrario también es cierto, aplicar técnicas positivas sin orientación ni validez social tampoco lo sería.

En definitiva, según el modelo ecológico-sistémico de Bronfenbrenner (1987), se podría decir que la línea técnica pertenece al microsistema mientras la teórica al macrosistema. El día a día, la interacción concreta, debe programarse técnicamente, es el microsistema. El ambiente, el clima, la cultura que rodea el sentido de la planificación pertenece al macrosistema. De esta manera el ACP es un modelo sistémico.

\section{Inicios del ACP}

\section{Antecedentes}

En un primer momento, el ACP es una reacción al abuso de las prácticas aversivas que se aplicaban a las personas con discapacidad intelectual y conductas problemáticas. Horner reconoce, explícitamente, que durante la década de los 80 surgió un movimiento alternativo al uso de las prácticas aversivas, fruto de las controversias teóricas sobre estas técnicas. Todo ello a la vez que aparece la defensa de los derechos de las personas con discapacidad para tener una vida digna y respetuosa, similar a la de cualquier otro miembro de la sociedad (Horner et al., 1990; Johnston, Foxx, Jacobson, Green y Mulick, 2006).

La denuncia de los abusos a personas vulnerables es histórica, ya en el siglo XVIII algunos psiquiatras defendieron un trato humano en los hospitales. Esta época ilustrada se conoce como la 'era moral' (Fariña López, 2011). Más próximo, en la década de los 80, un grupo de pedagogos de la Universidad de Nebraska defendieron el uso de técnicas educativas positivas, la enseñanza positiva, dirán, son todas aquellas prácticas y métodos que parten del respeto y la solidaridad humana, enfatizando la importancia de estos valores en los procesos terapéuticos y del cuidado (Jones y McCaughey, 1992).

Por otra parte, algunos importantes conductistas, a los que cabe suponer un profundo conocimiento de las técnicas, ya criticaron en sus inicios el uso de estímulos punitivos. Por ejemplo, Skinner decía que el castigo es una "reencarnación demoniaca”

(C) Ediciones Universidad de Salamanca

Siglo Cero, vol. 46 (2), n. ${ }^{\circ}$ 254, 2015, abril-junio, pp. 57-77 
(citado por Pelechano, 1980). En el Reino Unido Eysenck lo percibía como que “embrutece y conduce a conductas agresivas" (Eysenck, 1972: 99). Y algunos otros creen que es fácil pasar del castigo a "descargarse emocionalmente" (por ejemplo, Conklin, 1981; Pelechano, 1980).

También, el auge de la denominada Psicología Positiva sería otro antecedente del ACP. Martin Seligman, en la conferencia inaugural como presidente de la American Psychological Association defendió principios que lleven a los individuos y las comunidades hacia una vida plena (Seligman, 1998). Estos principios son coherentes, si no los mismos, que los defendidos por el ACP. Tamarit (1995), por ejemplo, señala que el enfoque de la psicología positiva trata de ayudar a las personas, en términos del aprendizaje permanente, para que disfruten su vida con la máxima independencia y posibilidades de resolver dificultades y limitaciones derivadas de su conducta.

Carr vio que la misión y el objetivo del ACP dependían de tres grandes temas: felicidad, amabilidad y optimismo, temas centrales de la Psicología Positiva. Carr ya declaraba esta alianza y la vio como una fuente de ideas para la evaluación e intervención en el ACP. En definitiva, tanto ACP como la Psicología Positiva se dirigen a cuestiones de calidad de vida: "La amplitud, profundidad y variedad que caracteriza a la Psicología Positiva y su evidente vinculación conceptual y práctica al Apoyo Conductual Positivo es una fuente de esperanza para nuestro campo y deja claro que no somos una voz solitaria en el desierto: somos una de las muchas voces de un coro" (Carr, 2007: 11).

Otro asunto es la relación entre ACP y ACA (Análisis conductual Aplicado), teoría y técnica. En general, quienes defienden el ACP reivindican la tecnología del ACA, cuya metodología trabaja con los principios científicos del empirismo. No obstante, la relación entre ambas líneas no siempre se valora de la misma manera. Para algunos autores, como Johnston et al. (2006), los conceptos más generales del ACP surgen y complementan la línea técnica. Es decir, todo lo que aparece bajo el paraguas ACP debe seguir los procedimientos del Análisis Conductual Aplicado. Sin embargo, para otros autores el ACP es una disciplina diferente del ACA, en el sentido de que el primero va más allá del simple sentido técnico, por ejemplo, Dunlap (2006) y Horner (2000) creen directamente que se trata de una materia autónoma. Sanetti, Dobey y Gritter (2012) piensan que el ACP está "en parte" fuera de la disciplina del ACA, llama la atención ese apelativo, en parte, que indica autonomía, por un lado, y dependencia, por otro. Al final atribuyen las técnicas de intervención y los procedimientos científicos a uno, al ACA, y un "más allá” al resto de asuntos que componen ACP. Sea como sea, la relación entre estas dos corrientes es un asunto pendiente y, por otra parte, se debe pensar que ambas incluso las técnicas no dejan de ser un ideal de cómo deberían ser las cosas (Singer y Wang, 2009).

En cualquier caso, como veremos más adelante, las relaciones entre el Análisis Conductual Aplicado y el Apoyo Conductual Positivo no están exentas de variadas y belicosas polémicas (Johnston et al., 2006).

Por último, hay que resaltar el término apoyo que alía el ACP con el 'paradigma del apoyo’ descrito por la Asociación Americana de Discapacidad Intelectual y del Desarrollo (Canal y Martín, 2002; Luckasson et al., 2002). 


\section{Actualidad}

Carr (1999) atribuye el nombre de ACP a Koegel, Koegel y Dunlap el año 1996. En esos momentos las preocupaciones se dirigen a tantear o unir el ACA con la línea teórica, es decir, con los valores adoptados en el mundo de la discapacidad intelectual (integración, calidad de vida, autodeterminación, etcétera). Un buen ejemplo para ver qué está cambiando lo comenta el autor al afirmar que una vez eliminado el comportamiento tratado no ha finalizado la intervención, ya que deberían plantearse cambios en el ambiente donde vive la persona. Esto es, ir más allá de la simple aplicación de una técnica y de sus resultados concretos, aun siendo positivos.

Por tanto, las características del ACP serán la elección de técnicas no aversivas, por un lado, y la insistencia en el cambio del tipo de vida, por otro. Por ejemplo, respetar las preferencias del individuo, las relaciones sociales y el acceso a situaciones comunitarias.

Entre las principales características una primera sería la utilización del análisis funcional de forma preventiva, es decir, de manera proactiva. Esto significa diseñar programas antes, o con independencia, de suceder los comportamientos a modificar. Carr, Levin, McConnachie, Carlson y Kemp (1996) lo indicaban directamente al decir que "el mejor momento para tratar una conducta es cuando no se da". Hoy se opta por una clasificación en tres divisiones, siguiendo el símil del semáforo: verde, ámbar y rojo. Las dos primeras son proactivas, la diferencia entre ellas radica en que en el ámbar se activan las pautas porque se observan precursores del comportamiento problemático.

La siguiente característica del ACP será la introducción de intervenciones multicomponente (LaVigna y Villis, 1992), indicando que éstas incluyen múltiples y simultáneas manipulaciones de diversas variables. Cambiar el estilo de vida es otra característica que también incluye intervenciones complejas diseñadas para aumentar las conductas positivas a la vez que se reducen las conductas no deseables (Koegel y Koegel, 1988).

Ahora bien, cuando se comenta la línea teórica ¿existe consenso?, ¿de qué aspectos se habla? Si se responde grosso modo no hay problema, se trata de la difusión de principios como: apoyo, autodeterminación, calidad de vida, etcétera. Es cuando se lee a diferentes autores que se observan problemas de precisiones claves y necesarias para establecer definiciones operativas y programas concretos.

Así, cada autor resalta una u otra dimensión, por ejemplo, Johnston et al. (2006) la idea educativa. El ACP es un enfoque integral que hace hincapié en la prevención de problemas del comportamiento a través de la programación educativa sistemática y cuidadosa.

Para Bambara y Kern (2005) el énfasis se situará en el enfoque de la resolución de problemas. Se ha de ser capaz de entender las razones de los problemas y diseñar, por tanto, las intervenciones integrales adaptadas a los motivos de estas dificultades. Todo ello pensando que se produce en un medio social, ambiental y cultural único.

Otros autores enfatizan el enfoque amplio del Apoyo Conductual Positivo, enfoque para la organización de apoyos, físicos, sociales, educativos y biomédicos necesarios

(C) Ediciones Universidad de Salamanca

Siglo Cero, vol. 46 (2), n. ${ }^{\circ}$ 254, 2015, abril-junio, pp. 57-77 
para alcanzar los objetivos básicos del estilo de vida deseado, al mismo tiempo que se irán reduciendo los problemas que impiden el logro del objetivo (Dunlap, Sailor, Horner y Sugai, 2009).

Hay quienes tratarán el ACP en el marco de un desarrollo eficaz de las intervenciones y programas para personas que muestran una conducta problemática, es decir, crear el ambiente idóneo donde los programas se desarrollarán (Anderson y Freeman, 2000).

En definitiva, unos autores resaltan o ponen el énfasis en los aspectos educativos, otros en el enfoque o marco, otros en el estilo de vida y otros en los derechos, aunque todos los autores coinciden en las características fundamentales del Apoyo Conductual Positivo. Así, con un repaso breve, se pueden hacer las siguientes descripciones donde se ven tanto las coincidencias como los diversos puntos de vista:

Carr (1998) en un artículo, traducido y publicado en Siglo Cero, describe las siguientes ideas con el apelativo de filosofía:

- El cliente como colaborador

- Prevención

- Modificación del contexto problemático y añade

- Una dimensión educativa

- Y una orientación sistémica

- Intervención multicomponente

- Cambio en el estilo de vida

- Perspectiva del ciclo vital

Horner et al. (1990) enfatizan los siguientes temas, referidos al ACP:

- Insistencia en cambiar el tipo de vida

- Análisis funcional

- Intervenciones complejas (multicomponente)

- Manipulación de circunstancias ecológicas y del entorno

- Insistencia en las manipulaciones de antecedentes

- Enseñanza de conductas adaptativas

- Creación de entornos con consecuencias eficaces

- Reducir al mínimo el uso de castigos

- Distinguir entre la emergencia y los programas proactivos

Por último, también señalan como elemento definitorio del ACP la inclusión de validez social para determinar la idoneidad de cualquier intervención.

Canal y Martín (2002) convergen con lo anterior, pero usan otros términos y añaden algunos:

- Global

- Múltiples intervenciones

- Enseñar habilidades alternativas (educación)

- Adaptar el ambiente (modificar el contexto)

- Considerar los valores de la persona

- Dignidad y preferencias 
- Estilo de vida

- Contextos de la vida diaria

- Disponibilidad de recursos

- Visión compartida

- Trabajar la conducta alternativa

Carr et al. (2002) recogen una terminología más clásica y agrupan todo ello en tres líneas: ABA (siglas en inglés), Normalizacion/Inclusión y Valores centrados en la persona y de ahí surgen nueve factores críticos:

- Cambios en la comprensión del estilo de vida

- Perspectiva del ciclo vital

- Validez ecológica

- Participación de los agentes (no sólo de expertos)

- Validez social

- Sistemas de Intervención Multicomponente

- Énfasis en la prevención

- Flexibilidad con relación a las prácticas científicas

- Perspectiva teórica múltiple

Por último, si acudimos a los cursos del sistema nacional de salud del país de Gales en uno de los documentos hablan de tres líneas maestras del ACP, igual que Carr, pero introducen la idea de Planificación Centrada en la Persona (PCP):

- ABA (Análisis Conductual aplicado, las técnicas)

- PCP (Plan centrado en la persona: visión de futuro)

- SRV (Considerar los valores sociales de las personas)

Como se ha indicado, si se adopta una perspectiva general y global todos mencionan las mismas líneas. Si la perspectiva es más concreta y matizada cambia. Esta puntualización puede no ser menor. Por ejemplo, si se introduce la perspectiva del ciclo vital, como hace Carr, el ACP se compromete durante toda la vida. En concreto, esto significa un compromiso para siempre con la persona, con los servicios y con los recursos.

Sobre el Plan Centrado en la Persona algunos no lo citan explícitamente y para otros es un eje central. Sin duda, cabe suponer que para todos los autores el PCP es un procedimiento básico. Por ejemplo, Carr, en otro artículo, indicará que se trata de la herramienta más útil del ACP para el establecimiento de objetivos relativos a la mejora de la calidad de vida (Carr, 2007).

Las tendencias que son incuestionables, es decir, irrenunciables que distinguen el Apoyo Conductual Positivo de cualquier otro modelo son, por ejemplo:

- La orientación preventiva

- Utilización de técnicas respetuosas y no aversivas

- El aprendizaje, apoyo e intervención de nuevas habilidades

- Intervenciones Multicomponentes 
- Utilización de procedimientos basados en la evidencia

- Resultados en estilo de vida y en concreto en Calidad de Vida

Sea como sea, no se ha llegado a un consenso sobre cuál es la definición de ACP (Dunlap, Kincaid, Horner, Knoster y Bradshaw, 2013).

Dunlap, de la Association for Positive Behavior Support (APBS), organización internacional fundada en 2003 en EE. UU. y dedicada a promocionar el ACP (http://apbs. $\mathrm{org} / \mathrm{main} . \mathrm{htm}$ ), está realizando una encuesta en Internet (https://www.surveymonkey. $\mathrm{com} / \mathrm{s} / \mathrm{PBSD}$ efinition) con el deseo de llegar a un consenso sobre este asunto. Mientras, la propia APBS lo define como un conjunto de estrategias basadas en la investigación, que se utilizan para aumentar la calidad de vida y disminuir los problemas de conducta, todo ello mediante la enseñanza de nuevas habilidades y la promoción de cambios en el medio ambiente de la persona.

La convergencia técnica-teórica es indiscutible y unánime. Una metáfora bastante mencionada señala que la técnica es ciega y la línea teórica indica la dirección correcta. Los valores no deben sustituir el empirismo, pero tienen que influir en el enfoque y la orientación de los esfuerzos. Singer y Wang (2009) hablan de un 'imperativo ético' descrito como análisis de la filosofía contemporánea sobre ideales y prohibiciones morales.

En definitiva, de cara a la formación de profesionales para aplicar ACP, deberían enseñarse tanto los principios ideológicos imperantes en el campo de la discapacidad intelectual como los procedimientos técnico-científicos. Entre los primeros habría normalización, integración, calidad de vida, planificación centrada en la persona, el paradigma de los apoyos o la autodeterminación. Y entre los segundos, refuerzo diferencial, habilidades, control de estímulos y técnicas de análisis funcional.

\section{Impacto}

El ACP ha sido aceptado ampliamente y hoy su aplicación se dirige a otros colectivos y ámbitos, además del de la discapacidad intelectual, por ejemplo, educación general, tercera edad y servicios juveniles de justicia. Los objetivos también se han ampliado al ambiente general de los colegios y servicios, ya no sólo a las conductas problemáticas.

Por ejemplo, Johnston et al. (2006), quienes comentan esta aceptación, citan al U.S. Departamento de Educación Nacional de Investigación y Rehabilitación en discapacidad (NIDRR), quien creó para el quinquenio 87-92 un consorcio de Universidades para la investigación de las técnicas no aversivas que abrió, a su vez, un centro de rehabilitación, investigación y entrenamiento (RRTC, por las siglas en inglés). Actualmente el centro se denomina RRTC-PBS (siglas en inglés) y su principal misión es difundir el Apoyo Conductual Positivo. El RRTC-PBS engloba a universidades de Oregón, Kansas, Kentucky, Misuri, Florida, Carolina del Norte y Florida Sur.

Swain-Bradway, Swoszowski, Boden y Sprague (2013) explican que el ACP está implantado en 18.000 escuelas en los Estados Unidos de América, con buenos

(C) Ediciones Universidad de Salamanca

Siglo Cero, vol. 46 (2), n. ${ }^{\circ}$ 254, 2015, abril-junio, pp. 57-77 
resultados sobre comportamientos problemáticos, tareas académicas y satisfacción escolar.

La existencia de publicaciones es otro elemento que describe la importante difusión del ACP. Debemos destacar en este sentido el Journal of Positive Behavior Interventions, creado en 1999.

Otro impacto lo demuestra que el ACP se plasme en Estados Unidos en leyes estatales, por ejemplo en el estado de Kansas y en leyes federales, especialmente en la de Educación de Personas con Discapacidad (IDEA) de 1997, como Intervenciones Conductuales Positivas.

Por último, es necesario destacar la existencia de la Association for Positive Behavior Support (APBS; http://www.apbs.org), de carácter internacional y dedicada a la promoción del ACP mediante jornadas internacionales anuales, además de organizar sus propios cursos, certificaciones y formación.

\section{Resultados}

Se pueden citar dos aproximaciones para conocer resultados que argumentan directa o indirectamente sobre ACP, los cualitativos y los cuantitativos.

Entre los resultados cualitativos comentar el trabajo de Bambara, Gomez, Koger, Lohrmann-ORourke y Xin (2001) sobre equipos humanos; el de Allen et al. (2011) de respuestas de servicios que aplican ACP, y una revisión de diez años de publicaciones en el Journal of Positive Behavior Interventions de Sanetti et al. (2012). Entre los cuantitativos se pueden comentar cuatro 'metaanálisis' que comparan los resultados de diversos procedimientos concretos de intervención: uno de Lennox, Miltenberger, Spengler y Erfanian (1988); otro de Carr, Robinson, Taylor y Carlson (1990); el siguiente de Didden, Duker y Korzilius (1997), y el de Carr (1999).

\section{Resultados cualitativos}

El primer estudio, de Bambara et al. (2001), compara las características que tienen los equipos que usan ACP para adultos que manifiestan conductas problemáticas severas con otros menos centrados en el ACP. Así, básicamente, hallaron que quienes tienen este modelo de trabajo:

a) Han asumido de forma clara los valores que les guían.

b) Cuentan con apoyos de otros equipos (por ejemplo de los técnicos).

c) Y saben que su misión es apoyar a la persona con discapacidad.

De esta manera, estos equipos piensan que las conductas problemáticas tienen razones lógicas para las personas que las manifiestan y, en su medida, las comprenden. Por tanto, ofrecen apoyos apropiados y respetuosos. Así, sus respuestas eran del tipo: estamos aquí por él o ella, los vemos como personas, etcétera. 
Los apoyos al equipo son percibidos como una lucha contra el estrés y el miedo que provocan las conductas problemáticas. Los equipos ejercen su derecho a ser oídos, es decir, a mantener discusiones internas y a dirimir los conflictos que van surgiendo. Conflictos que son tratados libremente a través de discusiones, en otras palabras, se intentan solucionar y, además, con consenso.

El trabajo de Allen et al. (2011), del país de Gales, es un proyecto para valorar la eficacia del ACP, mediante un cuestionario que mandan a distintos servicios. De los resultados recogidos (69\% de todos los cuestionarios remitidos) hay que destacar, entre otros, que se usan procedimientos diversos de evaluación e intervención (la mayoría, el 50\%, con planes individuales). El entrenamiento de los profesionales también es otro elemento clave (53\%). Un amplio uso de estrategias de prevención secundaria (antes de aparecer la conducta) como la distracción y la comunicación ( $84 \%$ y $63 \%$, respectivamente).

Los resultados efectivos sobre las conductas problemáticas son descritos como: daños a otros; a sí mismos; y al personal. Así, tras comparar la línea base con la de postintervención, observan una considerable reducción en todos los casos. Aumentan los usuarios que no provocan daños, por tanto, los servicios apenas tienen y desaparecen los más graves, calificados como muy serios.

En el trabajo de Sanetti et al. (2012) se recogen 72 estudios experimentales publicados en el Journal of Positive Behavior Intervention de 1999 a 2009. Un objetivo de los autores es determinar si los investigadores describen operativamente las variables independientes (los tratamientos) puesto que el ACP diseña intervenciones múltiples y generales que, si no se definen, corren el riesgo de introducir imprecisiones y confundir a los clínicos, quienes requieren descripciones fáciles y accesibles. Así, el 86,11\% de los estudios son operativos, sea a nivel operativo propiamente o referencial (un nivel inferior pero aceptable). No obstante, los datos revelan una tendencia decreciente a través del tiempo en la definición operativa y un aumento a nivel referencial. Para los autores la definición operativa significa que con su lectura sería fácil implementar una intervención similar y la referida más indirecta hace referencia a un manual, libro u otros, por lo tanto su implementación resulta menos obvia. El incremento de las referencias sobre las definiciones operativas son valoradas como una debilidad.

\section{Resultados cuantitativos}

Los metaanálisis son estudios que han ganado en popularidad desde que fueron descritos por primera vez en 1981. Se elaboran con normas propias y estrictas: QUOROM. Y, básicamente, permiten conocer los resultados de un campo concreto.

No obstante, los metaanálisis tienen serios problemas que conviene señalar como, por ejemplo, que cada uno elija sus propios criterios. Así, en algunos casos el éxito del tratamiento se valora tras modificar el $90 \%$ de la conducta, en otros cuando el $50 \%$ de los resultados no se solapan con los de la línea base. Las categorías de los tratamientos descritos tampoco coinciden, los hay con descripciones muy generales y otras muy minuciosas. Por último, deben mantenerse serias precauciones con las conclusiones

(C) Ediciones Universidad de Salamanca

Siglo Cero, vol. 46 (2), n. ${ }^{\circ}$ 254, 2015, abril-junio, pp. 57-77 
debido al baile de números y variables que se manejan, por ejemplo, un resultado bajo ciertas circunstancias puede que sea diferente bajo otras, por tanto, las cifras generales sólo son fotos fijas de una realidad dinámica.

El primer trabajo, de Lennox et al. (1988), puede describirse con la Tabla 1, un resumen que se ha diseñado con todos los resultados iguales o superiores al $75 \%$ de éxito y probados con un número de personas determinado (hay tratamientos descritos solamente a tres usuarios). El nivel 1 equivale a tratamientos respetuosos y positivos, mientras el 2 a neutros y el 3 a negativos.

\begin{tabular}{|c|c|c|c|}
\hline \multicolumn{4}{|c|}{$\begin{array}{l}\text { TABLA 1. Resultados descritos en el estudio de Lennox et al. (1988), } \\
\text { que superan el } 75 \% \text { de mejora y muestras }=>10\end{array}$} \\
\hline & Tratamiento & $\%$ de éxito & Sujetos \\
\hline Nivel 1 & $\begin{array}{l}\text { Cambio ambiental } \\
\text { Refuerzo } \\
\text { Rol playing }\end{array}$ & $\begin{array}{l}80 \\
81 \\
82 \\
\end{array}$ & $\begin{array}{l}13 \\
80 \\
14 \\
\end{array}$ \\
\hline Nivel 2 & Pantalla visual & 75 & 19 \\
\hline Nivel 3 & $\begin{array}{l}\text { Restricción mecánica } \\
\text { Spray }\end{array}$ & $\begin{array}{l}86 \\
82\end{array}$ & $\begin{array}{l}19 \\
10\end{array}$ \\
\hline & Promedio & 81 & 155 \\
\hline
\end{tabular}

Se halla que las técnicas positivas, nivel 1, mejoran de media el $81 \%$ de casos, el $75 \%$ con las denominadas intermedias y el $84 \%$ con las del nivel 3 . Luego las técnicas positivas son tan buenas como las restantes. En este trabajo cuando se toman todos los resultados (sin exclusión alguna) se hallan las siguientes cifras, respectivamente: 62,54 y $52 \%$, en este caso las técnicas positivas superan a las restantes.

Carr et al. (1990) fieles al ACP describen, sólo con técnicas positivas, la supresión de conductas problemáticas. A modo de resumen se diseña la Tabla 2 tomando una efectividad a partir del $90 \%$ de reducción (si se pasa al $80 \%$ de éxito los resultados aumentan en casi 20 puntos). Las técnicas de adquisición de habilidades resultan las más eficaces.

TABLA 2. Resultados -sólo técnicas positivas- descritos en el estudio de Carr et al. (1990), que superan el $90 \%$ de mej.

\begin{tabular}{|c|c|c|}
\hline Tratamiento & $\%$ de éxito & Sujetos \\
\hline Refuerzo Diferencial de Otras Conductas & 28 & 69 \\
\hline Refuerzo Diferencial de Conductas Incompatibles & 33 & 27 \\
\hline Adquisición de habilidades & 59 & 41 \\
\hline Control de estímulos & 48 & 54 \\
\hline Promedio & 42 & 191 \\
\hline
\end{tabular}

(C) Ediciones Universidad de Salamanca

Siglo Cero, vol. 46 (2), n. ${ }^{\circ}$ 254, 2015, abril-junio, pp. 57-77

$$
-67-
$$


Los resultados del tercer metaanálisis, de Didden et al. (1997), establecen la eficacia del procedimiento mediante el criterio de frecuencias no solapadas entre la línea base y el tratamiento. En la Tabla 3 se describen los principales resultados, así, realizar actividades obtiene un $100 \%$ de éxito, los refuerzos diferenciales de tasas bajas cuentan con un $99 \%$ y la desensibilización, un $97 \%$. En resumen, los datos permiten afirmar que frente a las técnicas aversivas las positivas compiten con igual éxito y eficacia, por tanto, pueden y deben elegirse con plenas garantías.

\begin{tabular}{|c|c|c|}
\hline \multicolumn{3}{|c|}{$\begin{array}{l}\text { TABLA 3. Resultados descritos en el estudio de Didden et al. (1997), } \\
\text { que superan el } 80 \% \text { de mejora }\end{array}$} \\
\hline Tratamientos positivos & \% de éxito & $\begin{array}{c}\text { Número de } \\
\text { comparaciones }\end{array}$ \\
\hline Actividad & 100 & 4 \\
\hline Entrenamiento en seguir órdenes $(*)$ & 92 & 10 \\
\hline Desensibilización $(*)$ & 97 & 8 \\
\hline Refuerzo diferencial de c. comunicativas $\left({ }^{*}\right)$ & 85 & 32 \\
\hline Refuerzo diferencial de tasas bajas & 99 & 8 \\
\hline Refuerzo diferencial con fichas & 83 & 21 \\
\hline Refuerzo diferencia sensorial & 89 & 11 \\
\hline Entrenamiento mediante guía del movimiento & 84 & 63 \\
\hline Práctica positiva & 88 & 50 \\
\hline \multicolumn{3}{|l|}{ Otros tratamientos } \\
\hline Estimulación auditiva negativa & 100 & 9 \\
\hline Castigo físico & 85 & 26 \\
\hline Olores desagradables & 94 & 7 \\
\hline Gusto desagradable & 82 & 27 \\
\hline Pantalla visual & 82 & 31 \\
\hline Extinción sensorial & 85 & 15 \\
\hline Supresión del movimiento & 100 & 8 \\
\hline Práctica negativa & 100 & 4 \\
\hline Refuerzo no contingente & 84 & 7 \\
\hline Medicamento: contra el apetito & 100 & 2 \\
\hline Medicamento: antidepresivo & 90 & 8 \\
\hline Medicamento: sal de litio & 100 & 1 \\
\hline
\end{tabular}

La Asociación Americana de Personas con Discapacidad Intelectual y del Desarrollo (Carr, 1999) edita en forma de libro el cuarto metaanálisis con una selección de investigaciones de calidad sólo con técnicas positivas. Los criterios de eficacia se miden en porcentajes de reducción entre la evaluación y el tratamiento. Los resultados se resumen 
escuetamente con la Tabla 4. De nuevo se demuestra la eficacia de las técnicas alineadas con ACP. En este caso, se valora el tratamiento directo con ACP en comparación con el uso de técnicas dirigidas al estímulo y las dirigidas al refuerzo. Los resultados no dejan lugar a dudas, las intervenciones con ACP son las que más reducción favorecen.

TABLA 4. Resultados en frecuencia descritos en el estudio de Carr et al. (1999)

\begin{tabular}{|l|c|c|c|}
\hline \multicolumn{1}{|c|}{ Tratamientos } & \multicolumn{3}{c|}{ Éxito } \\
\hline Basados en ACP & $100 \%$ & $90 \%$ & $80 \%$ \\
\hline Basados en técnicas dirigidas a los estímulos & 97 & 92 & 60 \\
\hline Basados en técnicas de refuerzo & 44 & 52 & 35 \\
\hline
\end{tabular}

En definitiva, estos metaanálisis demuestran que las técnicas positivas obtienen resultados igual o superiores a las negativas y, por tanto, si se debe elegir no hay dudas. En pocas palabras, las técnicas aversivas no se justifican a la hora de tratar conductas problemáticas.

\section{Críticas al ACP}

Los estudios que tratan ACP apenas recogen las posturas críticas, esto parece un error ya que la necesidad de responderlas favorecía el flujo de los argumentos sea en uno u otro sentido. Por otra parte, la ciencia debe ser abierta pues la distancia entre el conocimiento y la creencia es limitada. Muchas críticas hacia ACP son similares a las realizadas a la psicología positiva general. La revista Papeles del Psicólogo ha dedicado un monográfico y varios artículos a este asunto (Pérez, 2012; Poseck, 2006; Vázquez, 2013).

Una primera crítica consideraría que el ACP es un “mito” (Foxx, 2005) o una "Ilusión paternalista utópica” (Mulick y Butter, 2005). Brevemente, la mayoría de conceptos definidos desde la vertiente teórica serían ideas generales con escasa posibilidad de operativizar y por ende de aplicar los procedimientos experimentales.

Lógicamente, la crítica no significa ni debe entenderse como la defensa de la postura contraria a la criticada. Por ejemplo, quien no está en la sintonía del ACP no defiende la exclusión social en contraposición a la inclusión. Existe la tentación a pensar en esos términos y de hecho en ocasiones se cae en ese argumento, como una forma de acallar (desprestigiar) a quienes no favorecen la postura.

En las críticas de Foxx se describe el ACP como un movimiento que funciona con una fe ciega en las creencias ideológicas y una aceptación acrítica de las mismas. Todo ello acaba produciendo un "efecto narcótico" sobre los grupos (Foxx, 2005: 297). Por otra parte, este mito se alimenta de nuevas audiencias, que vienen de las “jóvenes 
generaciones' que aún no han apreciado la validación empírica y se dejan llevar por el atractivo del discurso ACP.

En segundo lugar, dirán estos autores, los estudios ACP no intervienen con conductas problemáticas muy graves y personas adultas. En el fondo sólo lo hacen con comportamientos ligeramente difíciles. Además, señalan que si se interviene en ambientes amplios el encarecimiento y la logística, asociados a la intervención, acaba siendo inviable; por ejemplo, Foxx (2005) comenta la existencia de programas con costos aproximados al millón de dólares.

En tercer lugar, la falta de autocrítica les lleva a pensar que cuando los programas fracasan se debe a que los servicios o los equipos no han aplicado los principios del ACP o bien que han faltado los apoyos necesarios. En definitiva, se entra en una espiral donde no falla el programa sino las trabas a la aplicación del ACP. Esta visión ha sido un tópico en nuestro entorno, Poplin (1991) en un artículo sobre falacias del campo de las discapacidades, publicado en la revista Siglo Cero, denunciaba lo mismo en otros términos: nunca falla el modelo (médico, educativo, etcétera), siempre es la persona o el entorno que no han sido valorados como debe ser.

En cuarto lugar, ponen en duda la validez del ACP porque las citas que realizan las principales figuras de este enfoque son endogámicas. Continuamente se citan entre sí, a sus propios libros, trabajos (incluidos aquellos sin publicar), conferencias y presentaciones. Crean sus propias revistas, lo que aumenta esta posibilidad endogámica. En contraposición a lo anterior, los autores inhiben cualquier controversia sobre técnicas aversivas. Los defensores del ACP simplemente ignoran todo aquello que no se refiere a su propia teoría o línea conceptual.

En quinto lugar, se cree que los líderes del ACP deben justificar un sistema menos técnico, puesto que sus intervenciones pueden (y deben) ser llevadas a cabo por cualquier figura: educadores, maestros, monitores, familias, etcétera, con independencia de sus credenciales. En esta línea, los autores críticos demandan investigaciones sobre estos cambios. A ello le contraponen los resultados contrastados de las técnicas basadas en las consecuencias de las conductas operantes.

En resumen, como indican Mulick y Butter (2005) la ciencia no es buena ni mala, lo malo es cuando se obtienen justificaciones de valores a través de la ciencia, como sucedió con el Darwinismo Social. Estos autores aceptan la idea de "nueva ciencia aplicada" cuando describen el ACP mediante el Análisis de Conducta Aplicado, a partir de ahí el resto es ideología o sistema de valores. La ciencia ya divide sus conocimientos en dos aspectos, que el ACP ignora: los estructurales de las cosas y los funcionales, así la parte teórica sería conocimiento funcional. En otras palabras, dirán, no se precisa acudir a discursos teóricos o ideológicos.

Mulick y Butter (2005) después de disertar sobre ciencia, ilusión y placebo concluyen que la palabra 'positivo' ejerce por sí misma un efecto ilusorio y contundente (al fin y al cabo, quién puede ir en contra de lo positivo). Los escritos sobre ACP acaban hablando de teoría y poco de ciencia. Añaden que los temas relacionados con técnicas aversivas ni los tratan sea en la dirección que sea, lo que priva a estudiantes y consumidores de un mínimo debate. En definitiva, dirán, los conceptos como normalización o inclusión son términos terapéuticos insuficientes y en cualquier caso ambos van detrás del tratamiento. 
Autores más próximos al ACA (Johnston et al., 2006) creen que la parte teórica del ACP, en línea con lo expuesto, es endeble. Al priorizar los valores, se excusa o filtra la investigación de los tratamientos, más allá de su eficacia. Tal énfasis puede guiar al clínico por la teoría, desentendiéndose o ignorando los resultados de las investigaciones.

Al final, se pone en juego la frontera de los tratamientos (no la bondad de la teoría) con relación al uso de técnicas no positivas. El ACP defendería, con relación a las técnicas aversivas, la ‘tolerancia cero'. Algunos críticos piensan que esto es un error ya que bajo ciertas circunstancias de peligro muy grave y tras la desesperanza de haber probado toda la batería de prácticas positivas debería ser posible contar con otros recursos. En cualquier caso, estos autores argumentan que los procedimientos excepcionales deberían tratarse con plenas garantías, permisos públicos, análisis exhaustivos, etcétera. Ahora bien, qué significa 'excepcional', a qué se refieren, cuál es el límite, todo ello no parece una cuestión definida.

Por último, como señalan Johnston et al. (2006) el ACP representa un intento bien intencionado de difundir un modelo de tratamiento basado en el análisis de la conducta. Sin embargo, dirán, se trata de un esfuerzo centrado e impulsado por la ideología, los intereses y su comercialización, más que por los resultados de la investigación y las consideraciones de los profesionales.

Sea como sea, el ACP ha sido un éxito como movimiento. Los resultados con técnicas e intervenciones positivas son iguales o superiores a las aversivas, como hemos visto en apartados anteriores. Los críticos plantean y creen que los aspectos teóricos son innecesarios, ideológicos y poco discriminativos, lo que no significa que vayan en contra de ellos, esto debe contemplarse como un desafío para ir mejorando. También, plantean si existen excepciones técnicas en los supuestos donde los daños de las propias conductas problemáticas son peores que los posibles remedios. Por último, como se ha indicado, piensan que el ACP no actúa realmente en situaciones dramáticas.

\section{Futuro del ACP}

Carr (2007) describe cuatro áreas de futuro: a) evaluación, b) intervención, c) formación y d) difusión a nuevos grupos.

En cuanto a la evaluación, desde la vertiente teórica, se deben introducir aspectos diversos como calidad de vida, integración, ciclo vital, agentes naturales, etcétera, luego parece evidente que se debe pasar de la evaluación microsistema, propia del Análisis Conductual, a la macrosistema propia de los entornos y teorías generales. Esto debe trabajarse desde una metodología múltiple, abierta, flexible y participativa. Las variables no son sólo las que hay en el entorno, también las que se podrían considerar como: expectativas, valores, apoyos posibles y aspectos directamente sugeridos por usuarios, las familias, educadores y servicios.

Lógicamente, todo ello conduce hacia los métodos cualitativos donde los indicadores y objetivos pasan por dimensiones como el consenso y las consideraciones personales,

(C) Ediciones Universidad de Salamanca

Siglo Cero, vol. 46 (2), n. ${ }^{\circ} 254,2015$, abril-junio, pp. 57-77 
por tanto, métodos tipo paneles, discusiones y dinámicas de grupos, los cuales vuelven a revalorizarse. Carr señala otras evaluaciones, por ejemplo, la de profesionales, la de contextos. Así, será motivo de valoración un aula, si hablamos del colegio; una unidad, si lo hacemos de un centro de día, o un hogar, en el caso de una residencia. Estos autores aconsejan indagar sobre herramientas, validez, fiabilidad y su aplicación por parte de los agentes naturales.

En este contexto, los expertos deberán aprender a tomar decisiones no sólo con otros expertos, sino con agentes que no necesariamente conozcan en profundidad estos temas. Por tanto, aprender a manejar ideas incluso de no ser del agrado de uno, esto significa habilidades de negociación.

La intervención, muy similar a la evaluación, pasará por el desarrollo de programas junto a los agentes naturales incluyendo círculos amplios como, según Carr, vecinos. En cuanto al lugar sucederá algo similar, de los lugares restrictivos se pasará a los naturales en sentido amplio, por ejemplo, lugares de vacaciones. Qué cambiar será otro aspecto donde se pasará del singular al plural, usando palabras de los autores, esto significa pasar de la conducta a las actividades que provoquen cambios en la calidad y el estilo de vida. Por último, el papel de la prevención frente a la reacción nos obliga al diseño de programas proactivos. Al hablar de prevención se va más allá: se refieren al desarrollo infantil, es decir, comenzar las intervenciones en la infancia temprana, de nuevo visión de ciclo vital.

El entrenamiento de las personas parece otra clave de futuro. Se deberá entrenar a los agentes naturales además de los profesionales. Los modelos irán más allá de los temarios cerrados y de las formas clásicas de enseñar ya que ACP es un modelo que representa la diversidad, no sólo una lista de técnicas y procedimientos. Los autores sugieren aprender de sucesos naturales y de situaciones 'en vivo'. Por supuesto, se deberá contar con las nuevas tecnologías.

Aquí, cabe mencionar el modelo de formación del servicio de salud del país de Gales, quienes trabajan con la plataforma moodle y ofrecen cursos 'on-line' con una estructura de módulos y temas muy claros y discriminativos. Éstos se dirigen a diferentes grupos de profesionales sean de salud, educación o servicios sociales y ofrecen títulos de diverso grado.

La difusión del ACP ya se observa en otros grupos, por ejemplo, en el sistema educativo general (no sólo en el de educación especial), en el juvenil-judicial y en el de tercera edad. Por otra parte, todo el campo de la discapacidad, con independencia de las conductas problemáticas, también se beneficia del ACP. En definitiva, se ha convertido en una visión activa sobre el trato positivo y respetuoso de todas las personas que, además, invita a trabajar con el enfoque, los valores y principios de los modelos de calidad de vida y del paradigma del apoyo, más allá de las conductas apoyadas o tratadas.

\section{Conclusiones}

Sin duda, la ciencia no es neutra y de alguna forma o en algún momento, como sucede a la hora de su aplicación, precisa valores que desempeñan un papel central. El

(C) Ediciones Universidad de Salamanca

Siglo Cero, vol. 46 (2), n. ${ }^{\circ}$ 254, 2015, abril-junio, pp. 57-77 
Apoyo Conductual Positivo defiende este papel, por tanto, su teoría no debe ser un simple añadido, más o menos formal. Al contrario, debe ser una suma a la comprensión racional del mundo social que nos rodea y a la posibilidad de acomodarlo a nuestra vida. De esta manera, la principal función de la teoría del ACP podría ser la de añadir valor a la vida.

Los valores actúan a través de un proceso de culturización que, a fuerza de aceptarlos y difundirlos, acaban convirtiéndose en un estilo de vida. Por ejemplo, algunos términos diagnósticos han llegado a ser malsonantes y se ha optado por no usarlos. Esto sucede aunque los términos no cambien la naturaleza y características del asunto que señalan. Para la ciencia empírica que sólo desea explicar la naturaleza "real" de las cosas, los términos sólo son símbolos que designan (en el lenguaje matemático esto es obvio). Sin embargo, si acudimos a las ciencias sociales vemos que los términos no son neutros ya que junto a su representación arrastran otras cuestiones ajenas, relacionadas con los valores. No obstante, puede ser lógico pensar que lo importante no son los conceptos (puras formas), sino las maneras concretas de hacer las cosas (el fondo de la cuestión). Las posturas siempre se balancean entre ambos polos, forma y fondo, sin una solución fácil y clara.

Así, el ACP es una cultura compartida pero en principio insuficiente, si sólo vemos la forma. A efectos de fondo, es decir, prácticos existe la necesidad de concretar, definir y operativizar los términos. Por ejemplo, la idea de 'ciclo vital': algunos autores la citan y en cambio otros no. Por poco que se vea, el ciclo vital introduce elementos significativos, introduce toda la vida. Por tanto, qué lugar e importancia tiene en el armazón teórico del ACP debería ser motivo para su desarrollo, más allá de ser citado por puro sentido común. En definitiva, los conceptos teóricos que caen dentro del ACP, su papel y jerarquía o relación entre ellos es un asunto abierto al cual conviene ir dando una forma definitiva y operativa. Ya que según como se traten los términos pueden ser conceptos científicos o una forma de hablar que todos comprenden y comparten. En resumen, podrían acabar siendo solamente un discurso de buenas intenciones.

Por otra parte, el ACP debería abandonar cierta visión de 'collage' donde caben todos los conceptos positivos, a veces poco discriminativos y sin duda incuestionables. Quien se acerca a su bibliografía obtiene esa visión. Carr (2007) lo veía así cuando comentaba que, si la herramienta es la Planificación Centrada en la Persona y los resultados deben darse en el marco de la Calidad de Vida, es necesario integrar todo ello mediante definiciones, indicadores y evaluación. Quizá, decantarse por varios términos estructurales podría ser una solución como por ejemplo calidad de vida, ciclo vital o PCP.

El excesivo y reiterado discurso, sin la referencia operativa, puede ser otro peligro en la credibilidad del ACP. Ciertas palabras, como positivo, valores, estilo de vida, etcétera, invitan a ese discurso. Hay documentos y escritos donde se puede apreciar esa réplica abundante. Por ejemplo, Sanetti et al. (2012) creen que los trabajos con ACP deben reforzar la definición operativa, piensan que en los mismos hay demasiadas descripciones generales e incluso programas que carecen de la posibilidad de ser replicados. 
En definitiva, existe el riesgo de aceptar los aspectos ideológicos, sin más, de no redoblar esfuerzos hacia los que tienen que ver con investigación, desarrollo, formación y cambio de actitudes. Esto no niega la dificultad, real, cuando los conceptos se amplían del microsistema al macrosistema, de aspectos técnicos a otros más teóricos. Ya se ha comentado que la Association for Positive Behavior Support (APBS) está realizando por Internet una encuesta sobre la definición del ACP, esta forma de trabajar permite conocer la visión del público, por tanto, aumenta la participación. Ahora bien, el alcance y uso científico de esta información, si se hace con esa intención, deberá realizarse con suma prudencia.

Como quiera que sea, el ACP es un movimiento aceptado por la mayoría, es ético y tiene pretensiones científicas. La vertiente técnica es operativa y trabaja con resultados y modelos cuasiexperimentales, más o menos estrictos. En este sentido, ya hay cuestiones claras, por ejemplo, elegir técnicas positivas porque son tan eficaces si no más que las aversivas. Es la línea teórica, con la misma pretensión científica, que requiere mayor precisión. Quizá, hay términos bastante desarrollados, como el de calidad de vida, donde la investigación es amplia. Ahora bien, por ahora la calidad de vida aparece en unos escritos ACP pero no en otros. Sin duda todos la aceptan y quienes no lo hacen explícito, posiblemente, pensarán que es tan obvio que no merece más explicaciones.

Este sentido científico obliga al ACP a contestar las críticas que recibe. Por ejemplo, la referida a la idea de que no trata a personas con comportamientos graves, peligrosos y resistentes. El riesgo que se corre, de no ser así, sería la merma de credibilidad. En definitiva, el ACP debe funcionar como una sociedad abierta. La comisión ACP de Baleares, por ejemplo, siendo partidaria de la tolerancia cero con relación a la estimulación aversiva abre debates sobre estas técnicas con la idea de hallar argumentos morales y técnicos conforme a la teoría defendida.

En nuestro país se pueden recomendar y consultar algunos escritos de interés como, por ejemplo, Canal (1998), en el ámbito educativo la revista Suports con trabajos de Sugai y Preciado (2009), Font y Castells (2009) y manuales como el de Canal y Martín (2002), Goñi, Martínez y Zardoya (2007). Meritorio el II Congreso Estatal sobre Alteraciones de Conducta (2013) organizado por la entidad Ampans en Manresa que nos permitió oír, entre otros, a los profesores George Sugai y Edwin Jones. No obstante, además del trabajo de describir y divulgar, sería deseable el de investigar. Entre otras cuestiones, responder sobre cuál es el grado de aplicación y desarrollo en los distintos servicios, es decir, su aceptación, conocimiento de los equipos, impacto sobre las personas, etcétera. También podría ser recomendable, como sucede en otros países, institucionalizar y avalar las acciones formativas para que no sean meros cursos informales sin una base que los cualifique, de forma que se garantice una mínima calidad.

Por último, el futuro del ACP debe desarrollarse desde la vertiente operativa como se ha descrito anteriormente. Es decir, la evaluación, la intervención, la formación y los resultados tanto en nuevos grupos que se benefician de este movimiento como en los grupos originales. Todo ello avalado por un sentido metodológico múltiple y amplio. Así, ya hemos visto en este trabajo estudios sobre intervenciones con conductas problemáticas, y los hay también sobre evaluación en escuelas (Singer y Wang, 2009),

(C) Ediciones Universidad de Salamanca

Siglo Cero, vol. 46 (2), n. ${ }^{\circ}$ 254, 2015, abril-junio, pp. 57-77 
en situación de justicia juvenil (Sprague et al., 2013), guías para responsables de servicios (Turnbull, Wilcox, Stowe y Turnbull, 2001) y diversidad cultural (Baker y George, 2013).

En definitiva, Apoyo Conductual Positivo trata de un movimiento de futuro que precisa ir, como no puede ser de otra manera, mejorando día a día y donde sólo podemos esperar resultados óptimos. Ahora bien, un enemigo contra el que cabe enfrentarse sería, en la línea de Sanetti et al. (2012), que no se convierta en una ideología vacía o en una teoría para contentar a un público externo, es decir, debería seguir siendo un reto científico.

\section{Referencias bibliográficas}

Allen, D., Lowe, K., Baker, P., Dench, C., Hawkins, S., Jones, E. y James, W. (2011). Assessing the effectiveness of positive behavioural support: The P-CPO Project. International Journal of Positive Behavioural Support, 1 (1), 14-23.

Anderson, C. M. y Freeman, K. A. (2000). Positive behavior support: Expanding the application of applied behavior analysis. The Behavior Analyst, 23 (1), 85-94.

Baker, C. y George, A. (2013). Positive Behavior Supports and Multicultural Concerns. Journal of Border Educational Research, 7 (1). Recuperado a partir de http://journals.tdl. org/jber/index.php/jber/article/view/7260.

Bambara, L. M., Gomez, O., Koger, F., Lohrmann-Orourke, S. y Xin, Y. P. (2001). More than Techniques: Team Members Perspectives on Implementing Positive Supports for Adults with Severe Challenging Behaviors. Research and Practice for Persons with Severe Disabilities, 26 (4), 213-228. DOI:10.2511/rpsd.26.4.213.

Bambara, L. M. y Kern, L. (2005). Individualized Supports for Students with Problem Behaviors: Designing Positive Behavior Plans. Guilford Press.

Bronfenbrenner, U. (1987). La ecología del desarrollo bumano. (Traducción de The ecology of human development: Experiment by nature and design. Cambridge: Harvard University Press, 1979). Madrid: Paidós.

Canal, R. (1998). El proceso para instaurar programas de apoyo conductual positivo en servicios residenciales. Siglo Cero, 29 (5), 11-22.

Canal, R. y Martín, M. V. (2002). Apoyo Conductual Positivo. Manuales de Trabajo en Centros de Atención a Personas con Discapacidad de la Junta de Castilla y León. Valladolid: Consejería de Sanidad y Bienestar Social. Junta de Castilla y León.

CArr, E. G. (1998). El apoyo conductual positivo: filosofía, métodos y resultados. Siglo Cero, 29 (5), 5-9.

Carr, E. G. (1999). Positive behavior support for people with developmental disabilities: a research synthesis. AAMR.

CARr, E. G. (2007). The expanding vision of positive behavior support: Research perspectives on happiness, Helpfulness, hopefulness. Journal of Positive Behaviour Interventions, 9 (1), 3-14.

Carr, E. G., Dunlap, G., Horner, R. H., Koegel, R. L., Turnbull, A. P., Sailor, W., ... Fox, L. (2002). Positive Behavior Support Evolution of an Applied Science. Journal of Positive Behavior Interventions, 4 (1), 4-16. Dor:10.1177/109830070200400102.

Carr, E. G., Levin, L., McConnachie, G., Carlson, J. I. y Kemp, D. C. (1996). Intervención comunicativa sobre los problemas del comportamiento. Madrid: Alianza Psicología.

(C) Ediciones Universidad de Salamanca

Siglo Cero, vol. 46 (2), n. ${ }^{\circ} 254,2015$, abril-junio, pp. 57-77 
Carr, E. G., Robinson, S., Taylor, J. C. y Carlson, J. I. (1990). Positive Approaches to the Treatment of Severe Behavior Problems in Persons with Developmental Disabilities: A Review and Analysis of Reinforcement and Stimulus-Based Procedures. (Monograph n. ${ }^{\circ}$ ). Seattle: The Association for Persons with Severe Handicaps.

Conklin, R. (1981). Cómo hacer que la gente haga cosas. Barcelona: Grijalbo.

Didden, R., Duker, P. C. y Korzilius, H. (1997). Meta-analytic study on treatment effectiveness for problem behaviors with individuals who have mental retardation. American Journal of Mental Retardation, 101 (4), 387-399.

Dunlap, G. (2006). The applied behaviour analytic heritage of PBS: A dynamic model of action-oriented research. Journal of Positive Behavior Interventions, 8, 58-60.

Dunlap, G., Kincaid, D., Horner, R. H., Knoster, T. y Bradshaw, C. P. (2013). A Comment on the Term "Positive Behavior Support". Journal of Positive Behavior Interventions. DOI:10.1177/1098300713497099.

Dunlap, G., Sailor, W., Horner, R. H. y Sugai, G. (2009). Overview and History of Positive Behavior Support. En W. Sailor, G. Dunlap, G. Sugai y R. Horner (Eds.), Handbook of Positive Behavior Support (pp. 3-16). Springer US. Recuperado a partir de http://link. springer.com/chapter/10.1007/978-0-387-09632-2_1.

Eysenck, H. J. (1972). Psychology is about people. Peguin Books Ltd.

FARIÑa López, E. (2011). La restricción física de los pacientes siglos xix y xx. Revista Rol de Enfermería, 34 (3), 182-189.

Font, J. y Castells, M. (2009). El pla educatiu de conducta: el segon nivell d'intervenció per al tractament de les conductes problemàtiques. Suports, 13 (1), 20-27.

Foxx, R. M. (2005). Severe Agressive and Self-Destructive Behavior: The Myth of the Nonaversive Treatment of Severe Behavior. En J. W. Jacobson, R. M. Foxx y J. A. Mulick (Eds.), Controversial Therapies for Developmental Disabilities: Fad, Fashion and Science in Professional Practices (pp. 295-310). New Jersey: Lawrence Erlbaum Associates.

Goñi, M. J., Martínez, N. y Zardoya, A. (2007). Apoyo Conductual Positivo. Algunas herramientas para afrontar las conductas difíciles. Madrid: FEAPS.

Horner, R. H. (2000). Positive behavior supports. Focus on Autism and other Developmental Disabilities, 15, 97-105.

Horner, R. H., Dunlap, G., Koegel, R. L., Carr, E. G., Sailor, W., Anderson, J., ... O’Neill, R. E. (1990). Toward a Technology of “Nonaversive” Behavioral Support. Journal of the Association for Persons with Severe Handicaps, 15 (3), 125-132.

Johnston, J., Foxx, R. M., Jacobson, J. W., Green, G. y Mulick, J. A. (2006). Positive Behavior Support and Applied Behavior Analysis. The Behavior Analyst, 29 (1), 51-74.

Jones, R. S. P. y McCaughey, R. E. (1992). Gentle teaching and applied behavior analysis: A critical review. Journal of Applied Behavior Analysis, 25, 853-867.

Koegel, R. L. y Koegel, L. K. (1988). Generalized responsivity and pivotal behaviors. En R. H. Horner, G. Dunlap y L. K. Koegel (Eds.), Generalization and maintenance: Lifestyle changes in applied settings (pp. 41-66). Baltimore: Paul H. Brookes.

Lavigna, G. W. y Villis, T. J. (1992). A Model for multielement treatment planning and outcome measurement. En D. E. Berkell (Ed.), Autism: Identification, Education, and Treatment. New Jersey: Lawrence Erlbaum Associates.

Lennox, D. B., Miltenberger, R. D., Spengler, P. y Erfanian, N. (1988). Decelerative Treatment Practices With Persons Who Have Mental Retardation: A Review of Five Years of Literature. American Journal on Mental Retardation, 92 (6), 492-501.

Luckasson, R., Borthwick-Duffy, S., Butinx, W. H. E., Coulter, D. L., Craig, E. M. (Рат), Reeve, A. y Tassé, M. J. (2002). Mental retardation: Definition, classification, and 
systems of supports (10th ed.) (vol. xiii). Washington, DC, US: American Association on Mental Retardation.

Mulick, J. A. y Butter, E. M. (2005). Positive Behavior Support: A Paternalistic Utopian Delusion. En J. W. Jacobson, R. M. Foxx y J. A. Mulick (Eds.), Controversial Therapies for Developmental Disabilities: Fad, Fashion and Science in Professional Practices (pp. 385404). New Jersey: Lawrence Erlbaum Associates.

Pelechano, V. (1980). Modelos básicos de aprendizaje. Valencia: Alfaplis.

Pérez, M. (2012). La psicología positiva: magia simpática. Papeles del Psicólogo, 33 (3), 183-201.

Poplin, S. (1991). La falacia reduccionista en las discapacidades para el aprendizaje: duplicación del pasado por reducción del presente. Siglo Cero, 137, 18-28.

Poseck, B. (2006). Psicología Positiva: Una nueva forma de entender la Psicología. Papeles del Psicólogo, 27 (1), 3-8.

SAnetTi, L. M. H., Dobey, L. M. y Gritter, K. L. (2012). Treatment Integrity of Interventions with Children in the Journal of Positive Behavior Interventions from 1999 to 2009. Journal of Positive Behavior Interventions, 14 (1), 29-46. DoI:10.1177/1098300711405853.

Seligman, M. E. P. (1998). The President's address. Annual report. (Annual report n. ${ }^{\circ}$ 4) (pp. 559-562). American Psychological Association.

Singer, G. H. S. y WANG, M. (2009). The Intellectual Roots of Positive Behavior Support and Their Implications for Its Development. En W. Sailor, G. Dunlap, G. Sugai y R. Horner (Eds.), Handbook of Positive Behavior Support (pp. 17-46). Springer US. Recuperado a partir de http://link.springer.com/chapter/10.1007/978-0-387-09632-2_2.

Sprague, J., Scheuermann, B., Wang, E., Nelson, C. M., Jolivette, K. y Vincent, C. (2013). Adopting and Adapting PBIS for Secure Juvenile Justicie Settings: Lessons Learned. The University of Oregon Institute on Violence and Destructive Behavior.

Sugai, G. y Preciado, J. (2009). L'estat actual i els avenços en el suport conductual positiu a nivell d'escola. Suports, 13 (1), 4-19.

Swain-Bradway, J., Swoszowski, N. C., Boden, L. J. y Sprague, J. R. (2013). Voices from the Field: Stakeholder Perspectives on PBIS Implementation in Alternative Educational Settings. Education and Treatment of Children, 36 (3), 31-46. Dor:10.1353/etc.2013.0020.

TAmarit, J. (1995). Conductas problemáticas y autismo: un analisis contextualizado. En La atención a alumnos con necesidades educativas graves y permanentes. Pamplona: Departamento de Educación, Cultura, Deporte y Juventud.

Turnbull, H. R., Wilcox, B. L., Stowe, M. y Turnbull, A. P. (2001). IDEA Requirements for Use of PBS Guidelines for Responsible Agencies. Journal of Positive Behavior Interventions, 3 (1), 11-18. DOI:10.1177/109830070100300103.

VÁzquez, C. (2013). La psicología positiva y sus enemigos: Una réplica en base a la evidencia científica. Papeles del Psicólogo, 34 (2), 91-115. 